\title{
An opportunity not to be spoiled
}

\author{
Research on nanotechnology applied to agriculture should focus on understanding the aspects in which the small \\ size can really make a difference.
}

T: he increasing world population could, in the next few decades, exacerbate an already pronounced unequal distribution of resources. Food security is one of the most pressing issues for governments to address. The second Sustainable Development Goal of the United Nations (https://go.nature.com/2LNpZHT), which aims at ending hunger, achieving food security, improving nutrition and promoting sustainable agriculture, is a direct demonstration of the political recognition of the urgency of this problem.

Achieving food security will require a range of social and economic actions. These include reducing food waste, improving the efficiency of irrigation systems, supporting local rural communities and tackling land degradation. Undoubtedly, scientific and technological innovation will also play an important role through increasing agricultural productivity.

As in many other fields, nanomaterials in particular could play a major part in increasing agricultural productivity, for example by enhancing crop resilience in adverse conditions and by improving the location and time of release of both nutrients and pesticides. Broadly speaking, we can imagine that the small size of nanomaterials combined with the high surface-area-tovolume ratio would allow the same effects as common fertilizers or pesticides to be obtained, but with a smaller amount of material.

In the Analysis by Melanie Kah and co-authors in this issue, the authors report a meta-analysis of the existing results on the efficiency of nanoagrochemicals, that is, nanofertilizers and nanopesticides, as well as on the potential risks they pose to the environment. A tangible conclusion of this effort is that nanoagrochemicals provide an overall improvement in efficiency of 20-30\% with respect to their non-nano counterparts. This result is certainly encouraging in itself. However, the perhaps more valuable feature of the article, especially for such a young research field, is the exposure of current shortcomings. One clear aspect that emerges is the scarcity of data comparing the efficacy and toxicity of nanoagrochemicals with conventional fertilizers and pesticides. Another fundamental point highlighted is that the majority of the existing results have been obtained in laboratory conditions, and

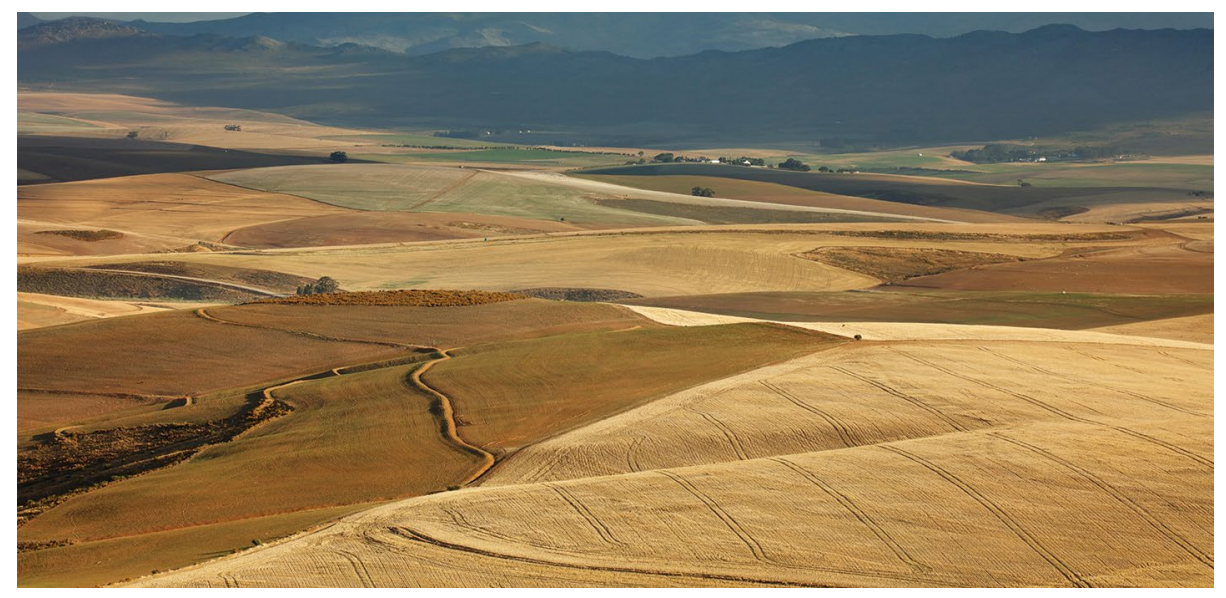

Credit: David Noton Photography / Alamy Stock Photo

there is a lack of advantages demonstrated under field conditions. Especially in terms of toxicity, more meaningful conclusions on the benefits of nanoagrochemicals can only be reached with larger-scale studies, in realistic agricultural conditions. Finally, there seems to be no comprehensive study of environmental effects that takes into consideration, for example, energy and water used. These types of studies will be essential to establish whether a nano-based approach to agriculture can be sustainable.

Another interesting feature emerging from the analysis is the comparison of the size distribution of nanoagrochemicals with the sizes used in regulations of nanomaterials. Sizes tend to vary according to whether they are measured by light scattering or by microscopy, and they are larger for nanopesticides than for nanofertilizers. Most importantly, however, they are often larger than $100 \mathrm{~nm}$, meaning that they would not be considered nanomaterials according to EU regulations - the EU definition establishes that in nanomaterials, at least $50 \%$ of the particles of an ensemble should be smaller than $100 \mathrm{~nm}$. This seems to suggest the necessity of reconsidering regulations to take into account the persistence of nano-effects for sizes larger than a specific threshold. In this sense, it is interesting to notice that the guidance for risk assessment of nanomaterials applied to food and agriculture, published by the European
Food Safety Authority on 4 July 2018, contemplates materials that may be larger than $100 \mathrm{~nm}$ but that retain properties typical of nanoparticles ${ }^{1}$.

Beyond regulations, understanding the relationship between size and properties is crucial. The Analysis by Kah et al. shows that only in a small portion of reports (11 out of the 78 examined) was a study of such a relationship directly investigated. The importance of such studies, highlighted also by Jason White and Jorge GardeaTorresdey in their News and Views article, is that in their absence it remains unclear whether the improvements observed in a new agrochemical formulation are indeed related to the inclusion of nanoparticles. It is true that broadly speaking the small size and high surface-area-to-volume ratio in nanoparticles can be beneficial. But using this justification without properly understanding the mechanisms of interaction between nanoparticles and crops, hence leading to the tailored design of new nanoagrochemicals, may in the long run undermine the potential of nanotechnology in agriculture, as has perhaps already happened in other fields.

Published online: 6 August 2018 https://doi.org/10.1038/s41565-018-0243-7

\section{References}

1. EFSA Scientific Committee. et al. EFSA J. 16, e05327 (2018). 